\title{
The convergence of Grandi's series
}

\author{
Open Mathematics Collaboration*† \\ January 10, 2020
}

\begin{abstract}
The infinite series $1-1+1-1+\ldots$ converges to $1 / 2$.
\end{abstract}

keywords: Grandi's series, convergence, Cesàro sum

\section{Introduction}

1. Infinitely many "divergent" series converge, depending on the summation method [1].

\section{Definition}

2. Let

$$
S_{n}=\sum_{i=0}^{n}(-1)^{n}=1-1+1-1+\ldots
$$

\section{Parity}

3. $(n$ is odd $) \rightarrow\left(S_{n}=1\right)$.

4. $(n$ is even $) \rightarrow\left(S_{n}=0\right)$.

*All authors with their affiliations appear at the end of this paper.

†Corresponding author: mplobo@uft.edu.br | Join the Open Mathematics Collaboration 


\section{Associativity}

5. $1+(-1+1)+(-1+1)+\ldots=1$

6. $(5) \equiv(n$ is odd $) \equiv(3)$

7. $(1-1)+(1-1)+\ldots=0$

8. $(7) \equiv(n$ is even $) \equiv(4)$

\section{Convergence}

9. Let

$$
S_{g}=\lim _{n \rightarrow \infty} S_{n}
$$

10. $S_{g}$ is known as the Grandi's series [2].

11. $S_{g}=1-1+1-1+\ldots$

12. $1-S_{g}=1-(1-1+1-1+\ldots)$

13. $1-S_{g}=1-1+1-1+\ldots$

14. $1-S_{g}=S_{g}$

15. $S_{g}=\frac{1}{2}$

\section{Final Remarks}

16. Since infinity is not a number, it is neither odd nor even.

17. From (3), (4), (16), $S_{g} \neq 1$, and $S_{g} \neq 0$.

18. Therefore,

$$
S_{g}=1-1+1-1+\ldots=\frac{1}{2} .
$$




\section{Open Invitation}

Review, add content, and co-author this article. Join the Open Mathematics Collaboration. Send your contribution to mplobo@uft.edu.br.

\section{Ethical conduct of research}

This original work was pre-registered under the OSF Preprints [3], please cite it accordingly [4]. This will ensure that researches are conducted with integrity and intellectual honesty at all times and by all means.

\section{References}

[1] Bromwich, Thomas John I'Anson. An introduction to the theory of infinite series. Macmillan and Company, limited, 1908.

[2] Kline, Morris. "Euler and infinite series." Mathematics Magazine 56.5 (1983): 307-315.

[3] COS. Open Science Framework. https://osf.io

[4] Lobo, Matheus P. "The Convergence of Grandi's Series." OSF Preprints, 4 Jan. 2020. https://doi.org/10.31219/osf.io/aq5xz

\section{The Open Mathematics Collaboration}

Matheus Pereira Lobo (lead author, mplobo@uft.edu.br) $)^{1,2}$

${ }^{1}$ Federal University of Tocantins (Brazil); ${ }^{2}$ Universidade Aberta (UAb, Portugal) 